\title{
Tannic Acid: Specific Form of Tannins in Cancer Chemoprevention and Therapy-Old and New Applications
}

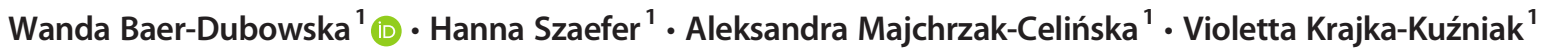

Published online: 27 March 2020

(C) The Author(s) 2020

\begin{abstract}
Purpose of Review This short review is aimed at providing an updated and comprehensive report on tannic acid biological activities and molecular mechanisms of action most important for cancer prevention and adjuvant therapy.

Recent Findings Tannic acid (TA), a mixture of digallic acid esters of glucose, is a common ingredient of many foods. The early studies of its anti-mutagenic and anti-tumorigenic activity were mostly demonstrated in the mouse skin model. This activity has been explained by its ability to inhibit carcinogens activation, as well as antioxidant and anti-inflammatory properties. Recently, the cell cycle arrest, apoptosis induction, reduced rate of proliferation, and cell migration and adhesion of several cancer cell lines as a result of TA treatment were described. The underlining mechanisms include modulation of signaling pathways such as EGFR/Jak2/STATs, or inhibition of PKM2 glycolytic enzyme. Moreover, epithelial-to-mesenchymal transition prevention and decrease of cancer stem cells formation by TA were also reported. Besides, TA was found to be potent chemosensitizer overcoming multidrug resistance. Eventually, its specific physicochemical features were found useful for generation of drug-loaded nanoparticles.

Summary TA was shown to be a very versatile molecule with possible application not only in cancer prophylaxis, as was initially thought, but also in adjuvant cancer therapy. The latter may refer to chemosensitization and its application as a part of drug delivery systems. More studies are required to better explore this subject. In addition, the effect of TA on normal cells and its bioavailability have to better characterized.
\end{abstract}

Keywords Tannic acid $\cdot$ Mouse skin model $\cdot$ Cancer cells $\cdot$ Chemosensitization $\cdot$ Nanomedicine

\section{Introduction}

Carcinogenesis is the process of transformation of a normal cell into a neoplastic one. This transition involves several steps starting with initiation, and followed by promotion and progression. Genetic and epigenetic changes caused by exogenous agents or endogenous factors are driving these stages, leading to the accumulation of mutations and epimutations in genes responsible for cellular homeostasis. Thus, cancer development involves gene-environment interactions. Moreover, oxidative stress and inflammation play important roles in carcinogenesis. Along with the epigenetic

This article is part of the Topical Collection on Redox Modulators

Wanda Baer-Dubowska

baerw@ump.edu.pl

1 Department of Pharmaceutical Biochemistry, Poznan University of Medical Sciences, Święcicki 4 Str, Poznań, Poland modifications, they cause aberrant expression of a variety of genes, both within the transforming cell population and the cells within the surrounding lesion [1].

Since carcinogenesis is a complex and long multistep process, intervention on its most early stages is considered as the most logical and promising approach of combating cancer. Chemoprevention is defined as the use of synthetic or natural substances to reverse, suppress, or prevent carcinogenic progression [2].

All of the above mentioned processes are targets for chemopreventive agents, and numerous reports of various bioactive foods and their extracted compounds, including tannins, have been shown to affect these hallmarks of carcinogenesis. Tannic acid (TA) is a specific tannin that formally contains 10 galloyl (3,4,5-trihydroxyphenyl) units surrounding a glucose center. However, commercially available and often naturally occurring TA consists of molecules with 2-12 galloyl moieties. TA contains no carboxyl groups, but is weakly acidic because of the multiplicity of phenolic hydroxyls. The hydroxyls also cause it to be extremely soluble in water [https://www.acs.org/content/ 
acs/en/molecule-of-the-week/archive/t/tannic-acid.html]. As the others tannins, TA is found in a wide range of plants, including fruits, green and black teas, nuts, and grains. However, TA major limitation in biological systems might be its relatively poor bioavailability. The available data indicate that following oral administration, $\sim 60 \%$ of tannic acid remains unchanged, but some are hydrolyzed to gallic acid by tannase in the intestine and are further metabolized to 4-Omethylgallic acid, pyrogallol, and resorcinol [3]. In vitro bioavailability of tannic acid was evaluated in ligated rat small intestine segments showing 50\% uptake, but not complete transfer through the gut wall [4]. One of the very first studies about TA date back to 1989, when TA was found to inhibit skin, lung, and forestomach tumors induced by chemical carcinogens [5••]. This activity was related to reduced carcinogens activation resulting from inhibition of specific forms of cytochrome P450, electrophile trapping, modulation of arachidonic acid metabolism [6,7], and ultimately inhibition of DNAadducts formation $[8,9 \bullet, 10]$. Similarly, to other polyphenols, TA possesses antioxidant activity [11]. However, it can act also as pro-oxidant resulting in oxidative DNA damage [12].

In the recent years, growing number of reports describe new mechanisms of TA activity and possible application not only in primary chemoprevention, but also in sensitization to conventional drugs used in anticancer therapy. Moreover, its specific physicochemical features showed up to be useful for nanomedicine purposes, including modern drug delivery systems (Fig. 1). This short review summarizes the current knowledge about TA chemopreventive activity and its possible application in cancer prophylaxis and adjuvant cancer therapy.

\section{Early Studies: Tannic Acid Affects the Processes Involved in Initiation and Promotion of Carcinogenesis in Mouse Skin Carcinogenesis Model}

The mouse skin model of multistage chemical carcinogenesis represents one of the best-established in vivo models for the study of the sequential and stepwise development of tumors. In addition, this model can be used to evaluate both novel skin cancer prevention strategies and the impact of genetic background and genetic manipulation on tumor initiation, promotion, and progression [13]. Therefore, the earliest data on the anticarcinogenic activity of TA were demonstrated in this model (Fig. 2).

In this regard, Muhtar et al. [14••] showed exceptional activity of TA, among naturally occurring plant phenols, in the protection against polycyclic aromatic hydrocarbons (PAH) 7,12-dimethylbenz[a]anthracene (DMBA), benzo[a]pyrene (B[a]P), 3-methylcholanthrene (3-MC), and direct carcinogen $N$-methyl- $N$-nitrosourea-induced skin tumorigenesis. The initiation of carcinogenesis by indirect carcinogens like $\mathrm{PAH}$ requires metabolic activation to ultimate carcinogenic form, which covalently binds to DNA. Cytochromes P450, mainly

Inhibition of cancer promotion and progression

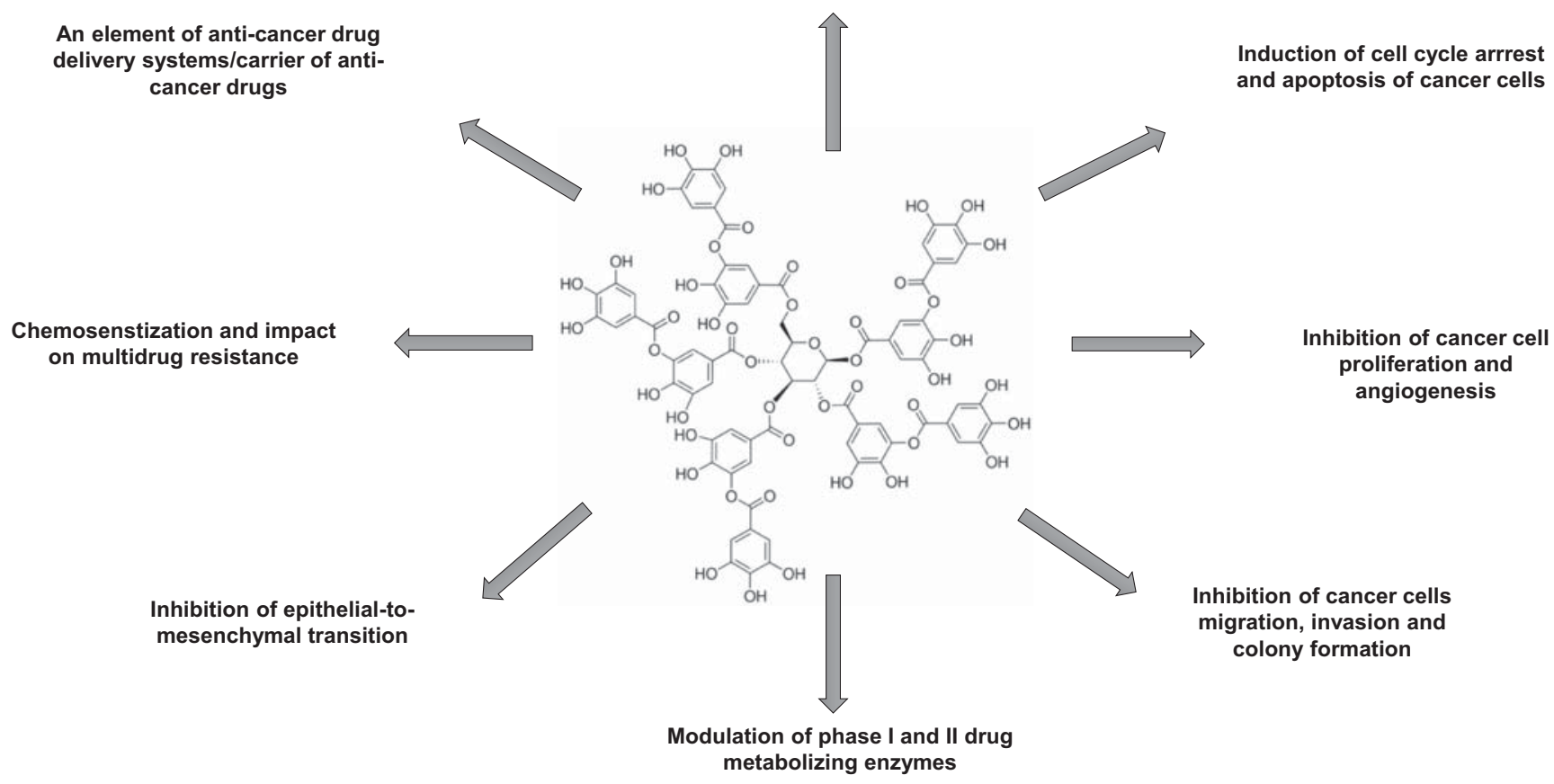

Fig. 1 The overview of mechanisms exerted by tannic acid important for cancer chemoprevention and/or therapy 


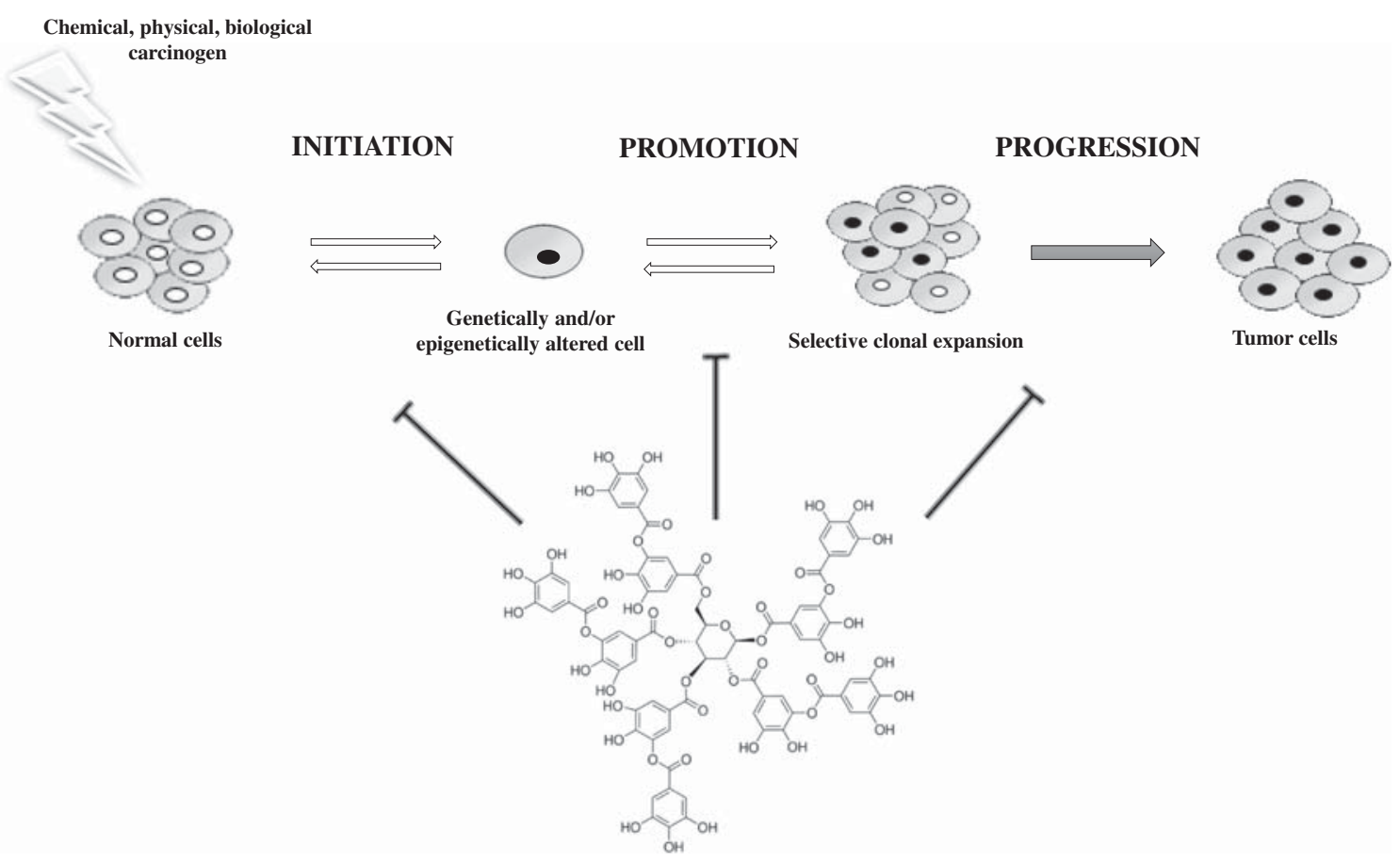

Fig. 2 Tannic acid can affect cancer initiation, promotion, and progression

CYP1A1, CYP1A2, CYP1B1, and CYP2E1, are involved in this process. Several studies including ours have shown the ability of TA to inhibit P450 monooxygenases or its specific isoforms [15-17]. Inhibitions of these enzymes led to the reduced carcinogen-DNA adduct formation in mouse epidermis $[8,18]$. Interestingly, analysis of DMBA-DNA adducts formed in vitro in the presence of 3-MC induced microsomes and in mouse epidermis showed almost complete inhibition of DMBA - dAdo adducts formation $[9,10]$. This was an important observation since the adenine adducts induced by ultimate DMBA metabolites (bay-region diol-epoxides) lead to mutation at the codon 61 of c-H-Ras and consequently initiate tumorigenesis in the mouse skin [19].

Moreover, modulation of phase II enzymes involved in ultimate carcinogens deactivation by TA was also observed both in animal models and in cell cultures in vitro. In animal models, this effect was to some extent tissue specific [16].

In tissue such as mouse epidermis, the role of modulation of enzymes involved in carcinogens activation and deactivation in the formation of DNA-adducts by TA depended on the type of carcinogen. While the reduction of B[a]P-DNA seems to result from decreased $\mathrm{B}[\mathrm{a}] \mathrm{P}$ activation, in case of DMBA-DNA adducts, the scavenging or masking of the binding sites to be occupied by DMBA reactive metabolites is more probable [15].

Following the initiation stage, the population of mutated cells is promoted to clonally expand during the second stage, referred to as "promotion." Promoting agents, which are both structurally and mechanistically diverse, stimulate cell signaling, increase production of growth factors, and generate oxidative stress and tissue inflammation [13]. Ours and the other research groups demonstrated that TA in the mouse epidermis stimulated by $12-O$-tetradecanoyphorbol-13-acetate (TPA) inhibited the activation of NF-KB transcription factor and subsequently expression and activity of inducible isoform of nitric oxide synthase (iNOS) and cyclooxygenase-2 (COX-2) enzymes - the key players of inflammation process [20]. As was mentioned above, TA through the reduction of DMBA, dAdo adducts may protect against Ras activation, which is required for chemical carcinogen-induced skin carcinogenesis in mice. Tumor promoter such as TPA expends the population of Ras initiated cells [13]. Thus, the compounds which affect the initiation of carcinogenesis in the mouse epidermis by reducing DNA adducts in critical genes like Ras family may inhibit the activation of NF-KB. The results of our studies demonstrated that TA may act in this way, i.e., by decreasing carcinogen-dAdo adducts formation, TA may inhibit NF- $\mathrm{KB}$ activation [20]. Moreover, TA affected also the activation of epidermal growth factor receptor (EGFR), activator protein-1 (AP-1) transcription factor, and signal transducers and activators of transcription (STATs) signaling pathways [21]. Moreover, in the same model, TA down-regulated the expression and activity of protein kinase $\mathrm{C}$ (PKC) which is thought to be a major intracellular receptor for the mouse skin tumor promoter TPA [22].

More recently, anti-promotional activity of TA in the mouse skin through the reduction of oxidative stress as well as COX-2, iNOS, PCNA (proliferating cell nuclear antigen) protein, and proinflammatory cytokine such as IL-6 release was confirmed [23]. Anti-inflammatory activity of TA was also shown in the context of house dust mite-induced atopic 
dermatitis. As the underlying mechanism of this activity, the induction of peroxisome proliferator-activated receptor $\operatorname{PPAR} \gamma$ protein was suggested [24•].

Chemopreventive activity of very low dose dietary TA administration in hepatoma bearing $\mathrm{C} 3 \mathrm{H}$ male mice was also demonstrated, but the studies on the mechanism of this activity in this model were not continued [25]. However, significant hepatoprotective effects against acetaminophen-induced hepatotoxicity were recently described [26]. The authors suggested that hepatoprotective mechanisms of TA may be related to antioxidation, anti-inflammation, and anti-apoptosis, thus the same mechanisms which might be responsible for TA chemopreventive activity observed in early study of hepatoma bearing mouse model.

\section{Tannic Acid Induces Cell Cycle Arrest, Apoptosis, and Limits Proliferation of Various Cancer Cells}

Promising data on the anticarcinogenic effect of TA in animal models, particularly in two-stage mouse skin model, stimulated the studies on the effect of TA on vital cellular processes such as apoptosis and proliferation in cancer cells of different origin. The observed effects of TA treatment often were celltype dependent. The examples of TA influence on cancer cell lines of different origin are presented below.

\section{Breast Cancer Cells}

Booth et al. [32] performed a series of experiments in which ER-positive breast cancer cells, MCF7, and triple negative $\left(\mathrm{ER}^{-}, \mathrm{PR}^{-}, \mathrm{HER}^{-}\right)$MDA-MB-231 along with nontumorigenic MCF10A were exposed to TA-cross-linked collagen type I beads. Growing cells remodeled collagen and released TA into surrounding medium leading to caspasemediated apoptosis. MCF7 cells were more sensitive to the pro-apoptotic effect of TA than MDA-MB-231. TA also induced apoptosis through the same mechanism in HER-2 positive cell line BT474 [27•, 28, 29].

Moreover, the same group successfully loaded adipocytes onto collagen type I beads with TA cross-linked. As adipocytes attached and grew on the TA cross-linked collagen beads, they remodeled the collagen, releasing TA, which then interacted with HER2 breast cancer cells, leading to its apoptosis. Viability assays also revealed the higher toxicity of TA to HER21 breast cancer cells as compared to normal human breast epithelial cells and adipocytes.

In contrast to the results of the above mentioned studies, no differences in the sensitivity to TA between MCF7 and MDAMB-231 breast cancer cells were observed in the investigation of Nie et al. [30]. One reason of this discrepancy might be the difference in TA concentrations applied in both studies which make the two approaches difficult to compare. This group also demonstrated that TA inhibits fatty acid synthase (FAS) activity. This key enzyme of fatty acids synthesis is overexpressed in human breast cancer cells. The authors suggested that inhibition of FAS may be one of the possible ways to induce apoptosis in these cells. Interestingly, TA showed higher cytotoxicity toward breast cancer cells than to FAS overexpressed 3 T3-L1 adipocytes. Thus, it is possible that in appropriate concentration, TA may induce apoptosis in cancer cells, but not in the surrounding adipocytes.

Moreover, TA has been reported to have high tyrosine kinase inhibition capacity. In this regard, strong inhibition of the tyrosine kinase activity of epidermal growth factor receptor (EGFR) and a weak inhibition of the P60 and insulin receptor tyrosine kinase were observed as a result of the treatment of human hepatoma HepG2 cells with TA. The molecular modeling study suggested that TA could be docked into the ATP-binding pockets of either EGFR or the insulin receptor [31]. EGFR-mediated phosphorylation of signal transducers and activators of transcription (STATs) leads to their activation. STATs can be activated also in EGFR-independent manner, involving phosphorylation by Janus kinases (JAKs). It was found that TA modulates the EGFR/Jak2/STAT3 pathway, inducing mitochondrial apoptosis in breast cancer cells lines: MCF-7, T47D, SK-BR 3, and MDAMB-231. Moreover, both the enhancement of STAT1 ser727 phosphorylation and the inhibition of STAT1 tyr701 phosphorylation were discovered as the key factors leading to G1 arrest upon TA treatment [32].

\section{Prostate Cancer Cells}

The effect of TA on proliferation, metastasis, and invasion was investigated in prostate cancer PC-3 and LNCaP cell lines, representing high and low metastatic potential, respectively. Treatment with TA significantly inhibited migration, invasion into matrigel, and ability to form colonies by prostate cancer cells. Modulation of the expression of cytochromes CYP17A1, CYP3A4, CYP2B6, and phase II enzymes NQO1, GSTM1, and GSTP1 was also observed in these cells [33].

\section{Head and Neck Cancer Cells}

The effect of TA on hypopharyngeal FaDu cancer cells and YD-38 gingival squamous cell carcinoma was investigated by Ta et al. [34] and Darvin et al. [35], respectively. In FaDu cells treatment with low dose $(25 \mu \mathrm{M})$ of TA led to cell cycle arrest in G2/M phase. As the dose of TA was increased, apoptosis was induced with the increase of cell population at sub-G1 phase. Both intrinsic and extrinsic cell death pathways were affected which was demonstrated by the evaluation of the expression of various cyclins and poly (ADP-ribose) polymerase (PARP) as well as phosphorylation of kinases of ERK, AKT and PKB [34]. In gingival squamous cell carcinoma 
YD-38 cells, TA inhibited Jak2/STAT3 pathway by preventing the expression as well as phosphorylation of its elements. It was also proved that TA exerted an intense activation of $p 21^{\text {Wafl/Cipl }}, p 27^{\text {Kip } 1}$, and $p 53$ genes confirming its role in G1 phase inhibition [35].

\section{Liver Cancer Cells}

The effect of TA on liver cancer cells was investigated in human hepatoma cell line HepG2. The results of our study showed activation of $\mathrm{Nrf2}$ /ARE signaling pathway as a result of the treatment with 2 and $10 \mu \mathrm{M}$ of TA. Subsequent induction of phase II enzymes, particularly GST, as well as antioxidant enzymes, was observed [36]. In contrast, recent study of Mhlanga et al. [37] showed increased levels of reactive oxygen species (ROS) and reactive nitrogen species [RNS] and down-regulation of antioxidant enzymes expression as a result of the treatment with $\mathrm{IC}_{50}$ and $\mathrm{IC}_{25}$ concentrations of TA, i.e., 29.4 and $14.7 \mu \mathrm{M}$, thus significantly higher than that applied in our study. The results of both studies confirm earlier suggestions [12] that TA may act as antioxidant or pro-oxidant depending on concentration. On the other hand, GSTT which was induced by TA in our study acts as a scavenger of electrophiles, such as epoxides. However, it may also metabolically activate halogenated compounds, thus producing a variety of intermediates that can potentially damage DNA and cells [36]. Therefore, its induction in cancer cells might be considered as ambiguous. Moreover, in another study, TA isolated from Caesalpinia coriaria induced G2/M phase cell cycle arrest and triggered cell death by microtubule stabilization in human hepatoma Hep3B cells [38]

\section{Colon Cancer Cells}

Interesting mechanism of inhibition of colorectal cancer cells (CRC) proliferation was proposed by Yang et al. [39••]. This group demonstrated that TA inhibits pyruvate kinase PKM2 activity and subsequently suppresses cell proliferation. They proposed, as an underlying mechanism of enzyme inhibition, binding of TA to lysine residue 433, which triggers the dissociation of PKM2 tetramers and blocks the activity of PKM2, not affecting PKM1 isozyme. The non-allosteric isoform PKM1 is constitutively active, and expressed in terminally differentiated tissues. By contrast, PKM2 is expressed in tissues with anabolic functions, and is subject to complex allosteric regulation. In the majority of cancer cells, the expression of PKM2 is increased, which suggests that PKM2 may be an attractive target for cancer therapy [40]. Therefore, TA might be considered as one of the molecules acting as PKM2 inhibitor.

\section{Glioma Cancer Cells}

The effect of TA was studied in rat C6 and human T98G glioma cell lines and verified in glioblastoma rat model. In our study, no significant differences in cell cycle distribution was observed in C6 glioma cells, but in T98G increased number of cells in $\mathrm{S}$ phase was found after incubation with TA at the concentrations lower and higher than $\mathrm{IC}_{50}$. In both cell lines, TA significantly increased the number of dead cells. Induction of apoptosis resulted mostly from increased level of caspase-3 [41]. In contrast, in the report of Bona et al. [42], the increased sub-G1 population of C6 cells, as a result of the treatment with TA in comparable concentrations, was described, along with the induction of apoptosis and necrosis. Moreover, TA reduced the formation and size of colonies, as well as cell migration and adhesion. Importantly, anti-glioma effect was also observed in vivo. TA decreased tumor volume and increased the area of intra-tumoral necrosis and infiltration of lymphocytes without damage of the surrounding tissue. These data suggest that TA may potentially support the therapy of these highly aggressive tumors.

As the examples above show, TA may inhibit proliferation and enhance, via different mechanisms, cell death of various cancer cells. However, so far, the similar data on normal cells or immortalized normal cells are scarce.

\section{The Cellular Effect of Tannic Acid Beyond Cancer Cell Death and Proliferation}

Epithelial-to-mesenchymal transition (EMT) is a dynamic, self-controlled, physiological process by which epithelial cells lose their junctional architecture and apical-basal polarity, detach from each other, and convert into a mesenchymal phenotype [43, 44]. EMT is crucial during embryogenesis, wound healing, and tissue regeneration; however, in noncontrolled conditions, it may lead to fibrosis, angiogenesis, and tumor progression with metastatic expansion [45]. It has recently been reported that TA treatment prevents TGF $\beta$-induced EMT in breast cancer cells as well as in lung epithelial cells $[46 \bullet \bullet, 47]$. The direct interaction between TA and TGF- $\beta 1$ was observed, attenuating the TGF- $\beta$ signaling [47]. In lung epithelial cells, TA also decreased the expression of $\mathrm{N}$ cadherin, type-1-collagen, fibronectin, and vimentin. Additionally, phosphorylation of Smad2 and 3, Akt, ERK1/2, JNK1/2, and p38 also decreased after the treatment with TA $[47,48]$. Moreover, in breast cancer cell line model, TA not only led to EMT inhibition, but also prevented the TGF $\beta$-induced increase in cancer stem cells (CSC) formation. Stemness-marker expression, including ALDH1 activity and the $\mathrm{CD} 44^{\mathrm{high}} / \mathrm{CD} 24^{\text {low }}$ ratio was also decreased after the treatment with only $10 \mu \mathrm{M}$ TA [46••]. Moreover, TA attenuated NF-KB signaling which is regarded as one of the most 
important mechanisms leading to the alleviation of CSC formation and EMT [46••]. In addition, blocking of NF- $\mathrm{BB}$ signaling by TA in bone marrow-derived macrophages inhibited NLRP3 inflammasome activation [49]. Recent data suggests that excessive NLRP3 inflammasome activation characterizes different cancer cells including head and neck squamous cell carcinoma and colorectal cancer cells. In U87 and GL261 xenograft mouse GBM model, NLRP3 inflammasome was involved in the resistance to radiotherapy [50]. Thus TA, by inhibiting NLRP3, may reduce cancer cell survival or improve the outcome of therapy.

The antiangiogenic properties of TA along with migration inhibition of MDA-MB-231 breast cancer cells were tested in the early study of Chen et al. [51]. TA inhibited cell migration induced by chemokine CXCL12. The effect of TA on the angiogenic consequences of CXCL12/CXCR4 interaction was studied using an in vitro assay of capillary tube outgrowth. Treatment with $0.5 \mathrm{~g} / \mathrm{ml}$ of TA completely inhibited tube formation induced by CXCL12, but not by basic fibroblast growth factor (bFGF) or endothelial cell growth supplement (ECGS) in bovine aortic endothelial cells (BAEC), suggesting that TA selectively antagonized the angiogenic activity of CXCL12. Chemokines, such as CXCL12 and their receptors, are now increasingly recognized as critical communication bridges between tumor cells and stromal cells to create a permissive microenvironment for tumor growth and metastasis [52]. Thus, both observations deserve further studies, but so far were not continued.

\section{Attempts to Apply Tannic Acid for Cancer Cells Sensitization and Overcoming Multidrug Resistance}

The treatment of cancer with chemotherapeutic agents has two major problems, time-dependent development of tumor resistance to therapy and nonspecific toxicity toward normal cells. A growing amount of studies indicate that plant polyphenols, including TA, are able to sensitize drug-resistant tumors to chemotherapy via various mechanisms, as well as to be protective from therapy-associated toxicities [53•]. One of the intracellular targets of polyphenols may be the proteasome. This proteolytic enzyme complex, responsible for intracellular protein degradation has been shown to play an important role in tumor growth and the development of drug resistance. Thus, inhibition of proteasome is considered as one of the mechanisms to overcome drug resistance and chemosensitize cancer cells to chemotherapy [54]. The ability of TA to inhibit the proteasome activity was tested and verified in purified $20 \mathrm{~S}$ proteasome and cellular $26 \mathrm{~S}$ proteasome in different cell types as well as in tumor-bearing mouse models. Inhibition of the proteasome function by TA resulted in increased p27 and Bax expression, and impaired cell cycle progression [55].
However, no combination with anticancer drugs was tested in this system.

Poly (ADP-ribose) glycohydrolase (PARG) is the main nuclear enzyme, which digests poly (ADP-ribose) into ADP-ribose. PARG inhibitors could also be considered as chemotherapeutic agents, because of its involvement in DNA repair [56]. TA was found to be PARG inhibitor, and through this mechanism, the sensitivity of ovarian carcinoma cells to cisplatin was increased. Combined treatment with TA and cisplatin induced apoptosis and increased DNA damage in the human ovarian carcinomas - cisplatin-resistant SKOV-3 CDDP/R cell line and cisplatinsensitive SKOV-3 cell line [57].

The main mechanism leading to the multidrug resistance (MDR) after the treatment with anticancer drugs is the overexpression of $\mathrm{ABC}$ transporters in cancer cells. Among $\mathrm{ABC}$ transporters, the major target of potential chemosensitizers is P-glycoprotein (P-gp; MDR-1) [58]. P-gp is expressed in various cancers and mediates MDR by actively transporting a wide range of anticancer drugs, including doxorubicin [59•]. Early report of Naus et al. [60] described interactions between TA and chemotherapeutic drugs in malignant human cholangiocytes. TA inhibited cellular efflux pathways, as determined by calcein retention assays by decreasing the expression of P-gp, MRP1, and MRP2 membrane efflux pumps. Modulation of drug efflux pathways resulted in synergistic effect to mitomycin $\mathrm{C}$ and 5-fluorouracil used in cholangiocarcinoma therapy.

In more recent study, the P-gp overexpressing human colon cancer cell line Caco-2 and human T-lymphoblastic leukemia cell line CEM/ADR 5000 were used to evaluate the effect of TA combination with doxorubicin (DOX). This combination synergistically sensitized both types of cells to the treatment. Decreased activity of P-gp as a result of the treatment with TA indicated that the inhibition of this protein is responsible for chemosensitization effect [58]. DOX is a highly effective drug, but its toxicity to normal cells, particularly, cardiomyocytes, restricts its therapeutic application. Thus, the use of phytochemicals as a protective tactic to reverse DOX-induced cardiotoxicity was the subject of several studies [61]. Zhang et al. [62] reported that pretreatment of rats with TA weakened DOX-induced cardiotoxicity by inhibiting oxidative stress, inflammation, and apoptotic damage. The possible protection of normal human oral keratinocytes against DOX-induced cytotoxicity without mitigating its cytotoxic potential against oral cancer cells was investigated in normal human oral keratinocytes and HSC-2 human oral squamous cell carcinoma cells. TA at the concentration above $50 \mu \mathrm{M}$ mitigated the DOX-induced keratinocyte toxicity without weakening DOX effect in SSC cells. In contrast, combination of TA at the concentration of $50 \mu \mathrm{M}$ and $100 \mu \mathrm{M}$ with DOX almost completely inhibited their survival [63]. The above data indicate that TA may be considered as a potential adjuvant in cancer chemotherapy. 


\section{Tannic Acid as a Carrier of Anticancer Drugs}

Nanomedical approaches to drug delivery aim at developing nanoscale particles or molecules to improve drug bioavailability both at specific places in the body and over a period of time. Different approaches have been applied to effectively load target drugs to enhance delivery resulting in increasing number of nanoparticles being carriers of anticancer drugs [64*0]. Recently TA attracted attention as a useful excipient for generation of drug-loaded nanoparticles. TA, being an additive molecule, can improve solubilization of various hydrophobic drugs intended for parenteral applications. TA possesses additive feature due to its low viscosity, good water solubility, and biocompatibility. TA can bind to drug molecules via hydrophobic interactions, which in turn form a self-assembled cross-linked network [67]. Such approach was used to generate TA - paclitaxel (PTX) selfassembly nanoparticles (TAP NPs) in order to potentiate PTX chemotherapeutic efficiency. The TAP NPs efficiently internalized into the cytoplasm of breast cancer MDA-MB-231 cells resulting in increased, in comparison with paclitaxel alone, inhibition of cells proliferation, clones formation, and migration. Moreover, TAP NPs increased beta-tubulin stabilization and apoptosis and limited P-gp-mediated drug efflux [65]. Moreover, the study of PTX administered in a form of PTX-loaded tannic acid/poly ( $N$-vinylpyrrolidone) nanoparticles (PTX-NP) showed that TA exhibits P-gp inhibitory function, whereas the intestinal retention of a drug is prolonged and trans-epithelial transport properties are improved. Oral administration of PTX-NP generated a high oral delivery efficiency and relative oral bioavailability of $25.6 \%$ in rats, and further displayed a significant tumorinhibition effect in a xenograft breast tumor model. These findings confirmed that PTX-NP might be a promising oral drug formulation for chemotherapy [66].

In another study, an injectable drug delivery system was developed, involving oxaliplatin (OXA) and TA incorporated into polymeric nanoparticles in a form of a thermo-sensitive hydrogel, (OXA/TA NPs-H). Intraperitoneal application of OXA/TA NPs$\mathrm{H}$ restricted the growth of CT26 peritoneal colon cancer in vivo, improved the quality of life, and prolonged the survival time of the model mice, which suggests this drug delivery system can be applied in colorectal cancer treatment [67].

Another form of nanoparticles with TA was formed by coassembling TA and polymer poly (2-oxazoline) and DOX as a model drug [68]. These polymeric nanoparticles showed high stability, good biocompatibility, and the cellular uptake. Thus, they can be considered as promising drug carriers for cancer therapy.

The major challenge in the design of anticancer drug carrier is the drug release in response to tumor-specific microenvironments (TMEs) such as low $\mathrm{pH}$, abnormal levels of ROS, and hypoxic conditions [69-72]. Hyaluronic acid, abundant in synovial fluid and the extracellular matrix, owing specific binding affinity for CD44-overexpressing cancer cells, has been used to prepare amphiphilic derivatives, capable of self-assembling into the nano-sized particles. However, these nanoparticles (HANPs) were unstable in physiological conditions and released a significant amount of drug into the bloodstream. This problem has been overcome by preparing metal $\left(\mathrm{Fe}^{3+}\right)$-phenolic (TA) network (MPN)-coated HANPs (MPNHANPs) as a pH-sensitive nano-carrier for hydrophobic drugs. DOX-loaded MPN-HANPs exhibited a higher cytotoxicity for the squamous cell carcinoma (SCC7), suggesting their potential use as a drug carrier in targeted cancer therapy $[73 \cdot \bullet]$.

Interesting application of TA in the treatment of lung cancer was described by Hatami et al. [74], who examined the TA interaction with the lung fluid (LF) - the major barrier for the distribution of drugs to the lungs. They demonstrated that TA binds to LF and forms self-assemblies, which profoundly enhance interaction with lung cancer cells. Thus, TA itself may be considered as a novel carrier for pharmaceutical drugs such as gemcitabine, carboplatin, and irinotecan. Therefore, TA, when used to formulate effective, yet nontoxic anticancer nanoparticles with drugs, has an excellent potential for translation from the bench to bedside cancer therapy.

\section{Conclusions}

TA showed up to be more versatile molecule than was initially thought. While the earliest investigations' concern was TA chemopreventive potential related to its ability to inhibit carcinogenesis initiation and promotion in animal models, over the years, the knowledge of its biological activities extended beyond this aspect. It was demonstrated that TA may interfere with the mechanisms, which might be important for cancer therapy, e.g. prevention of EMT or decrease in CSC formation. Moreover, available data indicate that TA may increase cancer cells sensitization to anticancer drugs and can help overcoming multidrug resistance. However, TA chemosensitization properties require more profound research on its effect on nontumorigenic cells. In addition, TA can be useful excipient for generation of drug-loaded nanoparticles. Therefore, TA certainly deserves further studies.

Funding Information This work is based upon work from COST Action NutRedOx-CA16112 supported by COST (European Cooperation in Science and Technology)

\section{Compliance with Ethical Standards}

Conflict of Interest The authors received no financial support in the writing of this manuscript.

Human and Animal Rights and Informed Consent This article does not contain any studies with human or animal subjects performed by any of the authors. 
Open Access This article is licensed under a Creative Commons Attribution 4.0 International License, which permits use, sharing, adaptation, distribution and reproduction in any medium or format, as long as you give appropriate credit to the original author(s) and the source, provide a link to the Creative Commons licence, and indicate if changes were made. The images or other third party material in this article are included in the article's Creative Commons licence, unless indicated otherwise in a credit line to the material. If material is not included in the article's Creative Commons licence and your intended use is not permitted by statutory regulation or exceeds the permitted use, you will need to obtain permission directly from the copyright holder. To view a copy of this licence, visit http://creativecommons.org/licenses/by/4.0/.

\section{References}

Papers of particular interest, published recently, have been highlighted as:

- Of importance

•- Of major importance

1. Hanahan D, Weinberg RA. Hallmarks of cancer: the next generation. Cell. 2011;144:646-74. https://doi.org/10.1016/j.cell.2011.02. 013.

2. Sporn MB. Approaches to prevention of epithelial cancer during the preneoplastic period. Cancer Res. 1976;36(7 PT 2):2699-702.

3. Nakamura Y, Tsuji S, Tonogai Y. Method for analysis of tannic acid and its metabolites in biological samples: application to tannic acid metabolism in the rat. J Agric Food Chem. 2003;51:331-9. https:// doi.org/10.1021/jf020847+.

4. Carbonaro M, Grant G, Pusztai A. Evaluation of polyphenol bioavailability in rat small intestine. Eur J Nutr. 2001;40:84-90. https:// doi.org/10.1007/s003940170020.

5.• Athar M, Khan WA, Mukhtar H. Effect of dietary tannic acid on epidermal, lung, and forestomach polycyclic aromatic hydrocarbon metabolism and tumorigenicity in SENCAR mice. Cancer Res. 1989;49:5784-8 This is one of the first articles describing chemopreventive activity of tannic acid.

6. Das M, Mukhtar H, Bik DP, Bickers DR. Inhibition of Epidermal Xenobiotic Metabolism in SENCAR Mice by Naturally Occurring Plant Phenols. Cancer Res. 1987;47:760-766.

7. Bartolí R, Fernández-Bañares F, Navarro E, Castellà E, Mañé J, Alvarez M, et al. Effect of olive oil on early and late events of colon carcinogenesis in rats: modulation of arachidonic acid metabolism and local prostaglandin E(2) synthesis. Gut. 2000;46:191-9. https:// doi.org/10.1136/gut.46.2.191.

8. Baer-Dubowska W, Gnojkowski J, Fenrych W. Effect of tannic acid on benzo [a]pyrene-DNA adduct formation in mouse epidermis: comparison with synthetic gallic acid esters. Nutr Cancer. 1997;29:4. https://doi.org/10.1080/01635589709514600.

9. Ignatowicz E, Balana B, Vulimiri SV, Szaefer H, Baer-Dubowska W. The effect of plant phenolics on the formation of 7,12dimethylbenz [a]anthracene-DNA adducts and TPA-stimulated polymorphonuclear neutrophils chemiluminescence in vitro. Toxicology. 2003;189:199-209. https://doi.org/10.1016/s0300$483 \times(03) 00148-3$ This article reports the ability of tannic acid to inhibit the formation of DMBA-dAdo adducts.

10. Szaefer H, Cichocki M, Brauze D, Baer-Dubowska W. Alteration in phase I and II enzyme activities and polycyclic aromatic hydrocarbons-DNA adduct formation by plant phenolics in mouse epidermis. Nutr Cancer. 2004;48:70-7. https://doi.org/10.1207/ s15327914nc4801_10.
11. Andrade RG Jr, Dalvi LT, Silva JM Jr, Lopes GK, Alonso A, Hermes-Lima M. The antioxidant effect of tannic acid on the in vitro copper-mediated formation of free radicals. Arch Biochem Biophys. 2005;437:1-9. https://doi.org/10.1016/j.abb. 2005.02.016.

12. Khan NS, Ahmad A, Hadi SM. Anti-oxidant, pro-oxidant properties of tannic acid and its binding to DNA. Chem Biol Interact. 2000;125:177-89. https://doi.org/10.1016/s0009-2797(00)001435.

13. Abel EL, Angel JM, Kiguchi K, DiGiovanni J. Multi-stage chemical carcinogenesis in mouse skin: fundamentals and applications. Nat Protoc. 2009;4:1350-62. https://doi.org/10.1038/nprot.2009. 120.

14.• Mukhtar H, Das M, Khan WA, Wang ZY, Bik DP, Bickers DR. Exceptional activity of tannic acid among naturally occurring plant phenols in protecting against 7,12-dimethylbenz(a)anthracene-, benzo(a)pyrene-, 3-methylcholanthrene-, and N-methyl-Nnitrosourea-induced skin tumorigenesis in mice. Cancer Res. 1988;48:2361-5 This the first report showing anti-carcinogenic activity of tannic acid.

15. Szaefer H, Krajka-Kuźniak V, Baer-Dubowska W. The effect of initiating doses of benzo [a] pyrene and 7,12-dimethylbenz [a] anthracene on the expression of PAH activating enzymes and its modulation by plant phenols. Toxicology. 2008;251:28-34. https://doi. org/10.1016/j.tox.2008.07.047.

16. Krajka-Kuźniak V, Baer-Dubowska W. The effects of tannic acid on cytochrome P450 and phase II enzymes in mouse liver and kidney. Toxicol Lett. 2003;143:209-16. https://doi.org/10.1016/ s0378-4274(03)00177-2.

17. Mikstacka R, Gnojkowski J, Baer-Dubowska W. Effect of natural phenols on the catalytic activity of cytochrome P450 2E1. Acta Biochim Pol. 2002;49:917-25.

18. Das M, Khan WA, Asokan P, Bickers DR, Mukhtar H. Inhibition of polycyclic aromatic hydrocarbon-DNA adduct formation in epidermis and lungs of SENCAR mice by naturally occurring plant phenols. Cancer Res. 1987;47:767-73.

19. Tang MS, Vulimiri SV, Viaje A, Chen JX, Bilolikar DS, Morris RJ, et al. Both (+/-)syn- and (+/-)anti-7,12-dimethylbenz [a]anthracene-3,4-diol-1,2-epoxides initiate tumors in mouse skin that possess -CAA- to -CTA- mutations at codon 61 of c-H-ras. Cancer Res. 2000;60:5688-95.

20. Cichocki M, Blumczyńska J, Baer-Dubowska W. Naturally occurring phenolic acids inhibit 12-O-tetradecanoylphorbol-13-acetate induced NF-kappaB, iNOS and COX-2 activation in mouse epidermis. Toxicology. 2010;268:118-24. https://doi.org/10.1016/j.tox. 2009.12.013.

21. Cichocki M, Dałek M, Szamałek M, Baer-Dubowska W. Naturally occurring phenolic acids modulate TPA-induced activation of EGFR, AP-1, and STATs in mouse epidermis. Nutr Cancer. 2014;66:308-14. https://doi.org/10.1080/01635581.2014.864419.

22. Szaefer H, Kaczmarek J, Rybczyńska M, Baer-Dubowska W. The effect of plant phenols on the expression and activity of phorbol ester-induced PKC in mouse epidermis. Toxicology. 2007;230:110. https://doi.org/10.1016/j.tox.2006.10.001.

23. Majed F, Rashid S, Khan AQ, Nafees S, Ali N, Ali R, et al. Tannic acid mitigates the DMBA/croton oil-induced skin cancer progression in mice. Mol Cell Biochem. 2015;399:217-28. https://doi.org/ 10.1007/s11010-014-2248-3.

24. Karuppagounder V, Arumugam S, Thandavarayan RA, Pitchaimani V, Sreedhar R, Afrin R, et al. Tannic acid modulates $\mathrm{NF} K \mathrm{~B}$ signaling pathway and skin inflammation in NC/Nga mice through PPAR $\gamma$ expression. Cytokine. 2015;76:206-13. https:// doi.org/10.1016/j.cyto.2015.05.016 This article reports the results of the studies showing the mechanism of antiinflammatory activity of tannic acid. 
25. Nepka C, Sivridis E, Antonoglou O, Kortsaris A, Georgellis A, Taitzoglou I, et al. Chemopreventive activity of very low dose dietary tannic acid administration in hepatoma bearing $\mathrm{C} 3 \mathrm{H}$ male mice. Cancer Lett. 1999;141:57-62. https://doi.org/10.1016/ s0304-3835(99)00145-7.

26. Zhang J, Song Q, Han X, Zhang Y, Zhang Y, Zhang X, et al. Multitargeted protection of acetaminophen-induced hepatotoxicity in mice by tannic acid. Int Immunopharmacol. 2017;47:95-105. https://doi.org/10.1016/j.intimp.2017.03.027.

27. Booth BW, Inskeep BD, Shah H, Park JP, Hay EJ, Burg KJ. Tannic acid preferentially targets estrogen receptor-positive breast cancer. Int J Breast Cancer. 2013;2013:369609. https://doi.org/10.1155/ 2013/369609 This is the first of the series of articles describing estrogen receptor depended effect of tannic acid.

28. Ngobili TA, Shah H, Park JP, Kwist KW, Inskeep B, Burg KJ, et al. Remodeling of tannic acid crosslinked collagen type I induces apoptosis in ER+ breast cancer cells. Anticancer Res. 2015;35:128590.

29. Jordan LG, Booth BW. HER2 ${ }^{+}$breast cancer cells undergo apoptosis upon exposure to tannic acid released from remodeled crosslinked collagen type I. J Biomed Mater Res A. 2018;106:26-32. https://doi.org/10.1002/jbm.a.36205.

30. Nie F, Liang Y, Jiang B, Li X, Xun H, He W, et al. Apoptotic effect of tannic acid on fatty acid synthase over-expressed human breast cancer cells. Tumour Biol. 2016;37:2137-43. https://doi.org/10. 1007/s13277-015-4020-z.

31. Yang EB, Wei L, Zhang K, Chen YZ, Chen WN. Tannic acid, a potent inhibitor of epidermal growth factor receptor tyrosine kinase. J Biochem. 2006;139:495-502. https://doi.org/10.1093/jb/mvj050.

32. Darvin P, Joung YH, Kang DY, Sp N, Byun HJ, Hwang TS, et al. Tannic acid inhibits EGFR/STAT1/3 and enhances p38/STAT1 signaling axis in breast cancer cells. J Cell Mol Med. 2017;21:720-34. https://doi.org/10.1111/jcmm.13015.

33. Karakurt S, Adali O. Tannic acid inhibits proliferation, migration, invasion of prostate cancer and modulates drug metabolizing and antioxidant enzymes. Anti Cancer Agents Med Chem. 2016;16: 781-9. https://doi.org/10.2174/1871520616666151111115809.

34. Ta LT, Nguyen TTK, Yoo H. Tannic acid-induced apoptosis in $\mathrm{FaDu}$ hypopharyngeal squamous cell carcinoma. Int J Oral Biol. 2019;44:43-9. https://doi.org/10.11620/IJOB.2019.44.2.43.

35. Darvin P, Baeg SJ, Joung YH, Sp N, Kang DY, Byun HJ, et al. Tannic acid inhibits the Jak2/STAT3 pathway and induces G1/S arrest and mitochondrial apoptosis in YD-38 gingival cancer cells. Int J Oncol. 2015;47:1111-20. https://doi.org/10.3892/ijo.2015. 3098.

36. Krajka-Kuźniak V, Paluszczak J, Szaefer H, Baer-Dubowska W. The activation of the Nrf2/ARE pathway in HepG2 hepatoma cells by phytochemicals and subsequent modulation of phase II and antioxidant enzyme expression. J Physiol Biochem. 2015;71:227-38. https://doi.org/10.1007/s13105-015-0401-4.

37. Mhlanga P, Perumal PO, Somboro AM, Amoako DG, Khumalo HM, Khan RB. Mechanistic insights into oxidative stress and apoptosis mediated by tannic acid in human liver hepatocellular carcinoma cells. Int J Mol Sci. 2019;20. https://doi.org/10.3390/ ijms20246145.

38. Sánchez-Carranza JN, Alvarez L, Marquina-Bahena S, Salas-Vidal E, Cuevas V, Jiménez EW, et al. Phenolic compounds isolated from Caesalpinia coriaria induce $\mathrm{S}$ and G2/M phase cell cycle arrest differentially and trigger cell death by interfering with microtubule dynamics in cancer cell lines. Molecules. 2017;22. https://doi.org/ 10.3390/molecules22040666.

39.• Yang P, Ding GB, Liu W, Fu R, Sajid A, Li Z. Tannic acid directly targets pyruvate kinase isoenzyme M2 to attenuate colon cancer cell proliferation. Food Funct. 2018;9:5547-59. https://doi.org/10. $1039 / \mathrm{c} 8$ fo $01161 \mathrm{c}$ This article proposes the interesting mechanism of the attenuation of cancer cell proliferation through inhibition of glycolytic enzyme.

40. Dong G, Mao Q, Xia W, Xu Y, Wang J, Xu L, et al. PKM2 and cancer: the function of PKM2 beyond glycolysis. Oncol Lett. 2016;11:1980-6. https://doi.org/10.3892/ol.2016.4168.

41. Zielińska-Przyjemska M, Kaczmarek M, Krajka-Kuźniak V, Łuczak M, Baer-Dubowska W. The effect of resveratrol, its naturally occurring derivatives and tannic acid on the induction of cell cycle arrest and apoptosis in rat C6 and human T98G glioma cell lines. Toxicol in Vitro. 2017;43:69-75. https://doi.org/10.1016/j. tiv.2017.06.004

42. Bona NP, Pedra NS, Azambuja JH, Soares MSP, Spohr L, Gelsleichter NE, et al. Tannic acid elicits selective antitumoral activity in vitro and inhibits cancer cell growth in a preclinical model of glioblastoma multiforme. Metab Brain Dis. 2019;26:283-93. https://doi.org/10.1007/s11011-019-00519-9.

43. Dongre A, Weinberg RA. New insights into the mechanisms of epithelial-mesenchymal transition and implications for cancer. Nat Rev Mol Cell Biol. 2019;20:69-84. https://doi.org/10.1038/ s41580-018-0080-4.

44. Antony J, Thiery JP, Huang RY. Epithelial-to-mesenchymal transition: lessons from development, insights into cancer and the potential of EMT-subtype based therapeutic intervention. Phys Biol. 2019;16:041004. https://doi.org/10.1088/1478-3975/ab157a.

45. Roche J. The epithelial-to-mesenchymal transition in cancer. Cancers (Basel). 2018;10. https://doi.org/10.3390/cancers10020052.

46.• Kim DA, Choi HS, Ryu ES, Ko J, Shin HS, Lee JM, et al. Tannic acid attenuates the formation of cancer stem cells by inhibiting NF$\mathrm{kB}$-mediated phenotype transition of breast cancer cells. Am J Cancer Res. 2019;9:1664-81 This article points out the possibility of attenuation of cancer stem cells formation by tannic acid through inhibition of ETM.

47. Pattarayan D, Sivanantham A, Krishnaswami V, Loganathan L, Palanichamy R, Natesan S, et al. Tannic acid attenuates TGF- $\beta 1$ induced epithelial-to-mesenchymal transition by effectively intervening TGF- $\beta$ signaling in lung epithelial cells. J Cell Physiol. 2018;233:2513-25. https://doi.org/10.1002/jcp.26127.

48. Avila-Carrasco L, Majano P, Sánchez-Toméro JA, Selgas R, LópezCabrera M, Aguilera A, et al. Natural plants compounds as modulators of epithelial-to-mesenchymal transition. Front Pharmacol. 2019;10:715. https://doi.org/10.3389/fphar.2019.00715.

49. Song D, Zhao J, Deng W, Liao Y, Hong X, Hou J. Tannic acid inhibits NLRP3 inflammasome-mediated IL-1 $\beta$ production via blocking NF- $\mathrm{KB}$ signaling in macrophages. Biochem Biophys Res Commun. 2018;503:3078-85. https://doi.org/10.1016/j.bbrc.2018. 08.096 .

50. Moossavi M, Parsamanesh N, Bahrami A, Atkin SL, Sahebkar A. Role of the NLRP3 inflammasome in cancer. Mol Cancer. 2018;17: 158. https://doi.org/10.1186/s12943-018-0900-3.

51. Chen X, Beutler JA, McCloud TG, Loehfelm A, Yang L, Dong HF, et al. Tannic acid is an inhibitor of CXCL12 (SDF-1alpha)/CXCR4 with antiangiogenic activity. Clin Cancer Res. 2003;9:3115-23.

52. Guo F, Wang Y, Liu J, Mok SC, Xue F, Zhang W. CXCL12/ CXCR4: a symbiotic bridge linking cancer cells and their stromal neighbors in oncogenic communication networks. Oncogene. 2016;35:816-26. https://doi.org/10.1038/onc.2015.139.

53. Garg AK, Buchholz TA, Aggarwal BB. Chemosensitization and radiosensitization of tumors by plant polyphenols. Antioxid Redox Signal. 2005;7:1630-47. https://doi.org/10.1089/ars.2005. 7.1630 This article provides a comprehensive review on chemo- and radiosensitization of tumor cells by plant polyphenols.

54. Shen M, Chan TH, Dou QP. Targeting tumor ubiquitin-proteasome pathway with polyphenols for chemosensitization. Anti Cancer Agents Med Chem. 2012;12:891-901. https://doi.org/10.2174/ 187152012802649978 . 
55. Nam S, Smith DM, Dou QP. Tannic acid potently inhibits tumor cell proteasome activity, increases p27 and Bax expression, and induces G1 arrest and apoptosis. Cancer Epidemiol Biomark Prev. 2001;10:1083-8.

56. Sasaki Y, Hozumi M, Fujimori H, Murakami Y, Koizumi F, Inoue $\mathrm{K}$, et al. PARG inhibitors and functional PARG inhibition models. Curr Protein Pept Sci. 2016;17:641-53. https://doi.org/10.2174/ 1389203717666160419145130.

57. Sun Y, Zhang T, Wang B, Li H, Li P. Tannic acid, an inhibitor of poly (ADP-ribose) glycohydrolase, sensitizes ovarian carcinoma cells to cisplatin. Anti-Cancer Drugs. 2012;23:979-90. https://doi. org/10.1097/CAD.0b013e328356359f.

58. Li H, Krstin S, Wink M. Modulation of multidrug resistant in cancer cells by EGCG, tannic acid and curcumin. Phytomedicine. 2018;50:213-22. https://doi.org/10.1016/j.phymed.2018.09.169.

59. Khan M, Maryam A, Mehmood T, Zhang Y, Ma T. Enhancing activity of anticancer drugs in multidrug resistant tumors by modulating P-glycoprotein through dietary nutraceuticals. Asian Pac J Cancer Prev. 2015;16:6831-9. https://doi.org/10.7314/apjcp.2015. 16.16.6831 This article presents the mechanism of overcoming MDR by nutraceuticals.

60. Naus P, Henson R, Bleeker G, Wehbe H, Meng F, Patel T. Tannic acid synergizes the cytotoxicity of chemotherapeutic drugs in human cholangiocarcinoma by modulating drug efflux pathways. J Hepatol. 2007;46:222-9. https://doi.org/10.1016/j.jhep.2006.08. 012.

61. Xiao J, Sun GB, Sun B, Wu Y, He L, Wang X, et al. Kaempferol protects against doxorubicin-induced cardiotoxicity in vivo and in vitro. Toxicology. 2012;292:53-62. https://doi.org/10.1016/j. tox.2011.11.018.

62. Zhang J, Cui L, Han X, Zhang Y, Zhang X, Chu X, et al. Protective effects of tannic acid on acute doxorubicin-induced cardiotoxicity: involvement of suppression in oxidative stress, inflammation, and apoptosis. Biomed Pharmacother. 2017;93:1253-60. https://doi. org/10.1016/j.biopha.2017.07.051.

63. Sheng H, Ogawa T, Niwano Y, Sasaki K, Tachibana K. Effects of polyphenols on doxorubicin-induced oral keratinocyte cytotoxicity and anticancer potency against oral cancer cells. J Oral Pathol Med. 2018;47:368-74. https://doi.org/10.1111/jop.12685.

64.• Chowdhury P, PKB N, Hatami E, Wagh S, Dan N, Tripathi MK, et al. Tannic acid-inspired paclitaxel nanoparticles for enhanced anticancer effects in breast cancer cells. J Colloid Interface Sci. 2019;535:133-48. https://doi.org/10.1016/j.jcis.2018.09.072 This article reports the results of a comprehensive study on tannic acid as excipient of anti-cancer drug-paclitaxel.
65. Chen YN, Jiao C, Zhao Y, Zhang J, Wang H. Self-assembled polyvinyl alcohol-tannic acid hydrogels with diverse microstructures and good mechanical properties. ACS Omega. 2018;3:11788-95. https://doi.org/10.1021/acsomega.8b02041.

66. Le Z, Chen Y, Han H, Tian H, Zhao P, Yang C, et al. Hydrogenbonded tannic acid-based anticancer nanoparticle for enhancement of oral chemotherapy. ACS Appl Mater Interfaces. 2018;10:4218697. https://doi.org/10.1021/acsami.8b18979.

67. Ren Y, Li X, Han B, Zhao N, Mu M, Wang C, et al. Improved anticolorectal carcinomatosis effect of tannic acid co-loaded with oxaliplatin in nanoparticles encapsulated in thermosensitive hydrogel. Eur J Pharm Sci. 2019;128:279-89. https://doi.org/10.1016/j. ejps.2018.12.007.

68. Liu F, Kozlovskaya V, Zavgorodnya O, Martinez-Lopez C, Catledge S, Kharlampieva E. Encapsulation of anticancer drug by hydrogen-bonded multilayers of tannic acid. Soft Matter. 2014;10: 9237-47. https://doi.org/10.1039/c4sm01813c.

69. Wang S, Huang P, Chen X. Stimuli-responsive programmed specific targeting in nanomedicine. ACS Nano. 2016;10:2991-4. https:// doi.org/10.1021/acsnano.6b00870.

70. Son S, Deepagan VG, Shin S, Ko H, Min J, Um W, et al. Ultrasmall gold nanosatellite-bearing transformable hybrid nanoparticles for deep tumor penetration. Acta Biomater. 2018;79:294-305. https:// doi.org/10.1016/j.actbio.2018.08.019.

71. Deepagan VG, Kwon S, You DG, Nguyen VQ, Um W, Ko H, et al. In situ diselenide-crosslinked polymeric micelles for ROS-mediated anticancer drug delivery. Biomaterials. 2016;103:56-66. https:// doi.org/10.1016/j.biomaterials.2016.06.044.

72. Thambi T, Park JH, Lee DS. Hypoxia-responsive nanocarriers for cancer imaging and therapy: recent approaches and future perspectives. Chem Commun (Camb). 2016;52:8492-500. https://doi.org/ $10.1039 / \mathrm{c} 6 \mathrm{cc} 02972 \mathrm{~h}$.

73.• Shin JM, Choi GH, Song SH, Ko H, Lee ES, Lee JA, et al. Metalphenolic network-coated hyaluronic acid nanoparticles for $\mathrm{pH}$ responsive drug delivery. Pharmaceutics. 2019;11. https://doi.org/ 10.3390/pharmaceutics11120636 This article reports tannic acid application in $\mathrm{pH}$ responsive drug delivery.

74. Hatami E, Nagesh PKB, Chowdhury P, Chauhan SC, Jaggi M, Samarasinghe AE, et al. Tannic acid-lung fluid assemblies promote interaction and delivery of drugs to lung cancer cells. Pharmaceutics. 2018;10. https://doi.org/10.3390/pharmaceutics10030111.

Publisher's Note Springer Nature remains neutral with regard to jurisdictional claims in published maps and institutional affiliations. 